\section{The Fischer Indole Synthesis}

THE recently suggested theory of Pausacker and Schubert ${ }^{1}$ involving free radicals in the Fischer indole synthesis appeared to us improbable. It seemed more likely that these experimental results could be explained quite readily by hydrolyses of the hydrazones and recombination to produce two new hydrazones. It may be remarked here that a fifth product, 1:8-dimethyl carbazole, should also have been isolated in small yield in the case quoted. The possibility that this is the correct interpretation is admitted by Pausacker in a second communication ${ }^{2}$. Evidence has now been obtained which shows clearly that it is the correct one, and that the free radical theory is untenable. The experimental results may be summarized as follows :

(1) Acetone phenylhydrazone (1 mol.), cyclohexanone (1 mol.) for $\frac{1}{2}$ hour, yield tetrahydrocarbazole (50 per cent).

(2) Cyclohexanone 2:4-dinitrophenylhydrazone (1 mol.), phenylhydrazine ( $1 \mathrm{~mol}$.) for 24 hours, yield tetrahydrocarbazole (18 per cent), $2: 4$-dinitrophenylhydrazine (31 per cent).

(3) Cyclohexanone 2 : 4-dinitrophenylhydrazone (1 mol.), acetone phenylhydrazone $(5 \cdot 3 \mathrm{~mol}$.) for 32 hours, yield tetrahydrocarbazole (16 per cent).

(4) Benzaldehyde phenylhydrazone (1 mol.), cyclohexanone (1 mol.) for 25 hours, yield tetrahydrocarbazole (5 per cent).

All reactions were carried out in boiling glacial acetic acid. It will be recalled that under these conditions acetone phenylhydrazone and cyclohexanone 2:4-dinitrophenylhydrazone do not cyclize. Experiments (1), (2) and (4) show clearly that hydrolysis and recombination to a new hydrazone occurred, and (3) is particularly significant in this respect. The isolation of dinitrophenylhydrazine in (2) excludes completely the possibility of a free radical mechanism. Moreover, the yields of tetrahydrocarbazole in each experiment are approximately those to be expected on the hydrolysis-recombination theory.

There still remains the possibility that the cyclization itself proceeds by a free-radical mechanism; but several strong arguments against this theory can be advanced. By careful working, a yield of more than 95 per cent of tetrahydrocarbazole can be obtained from the cyclization of cyclohexanone phenylhydrazone by boiling glacial acetic acid. It is difficult to believe that this product would be formed in such high yield if free radicals were involved. Secondly, free $\mathrm{C}_{6} \mathrm{H}_{5} \mathrm{NH} \cdot$ radicals would surely combine to give some hydrazobenzene, which under the reaction conditions would be converted to benzidine. Tests which detect $0.00003 \mathrm{gm}$. of benzidine gave negative results when applied to the appropriate fraction from the cyclization of $40 \mathrm{gm}$. of cyclohexanone phenylhydrazone. Finally, any free radicals would be expected to react mainly with the solvent by the reaction

$$
X \cdot+\mathrm{CH}_{3} \mathrm{COOH} \rightarrow \mathrm{CH}_{3} \mathrm{COO} \cdot+\mathrm{XH},
$$

which would be followed by

$$
\mathrm{CH}_{3} \mathrm{COO} \cdot \rightarrow \mathrm{CH}_{3} \cdot+\mathrm{CO}_{2} ;
$$

and hence large amounts of carbon dioxide should be liberated. It was found, however, that the yield of carbon dioxide was only 0.4 per cent. This probably arises from two other sources: (1) a small part coming from a side-reaction of unknown nature, since acetone phenylhydrazone slowly evolves carbon dioxide on boiling with glacial acetic acid, and (2) the major part from the thermal decomposition of acetic acid. The reasons for this suggestion are, first, that carbon dioxide is evolved rapidly just as the mixture reaches the boiling point, and secondly, the reaction is strongly exothermic and there may be present isolated points of high energy content.

P. H. GORE

G. K. Hughes

E. RITCHIE

School of Chemistry,

University of Sydney. June 16.

${ }^{1}$ Nature, 163, 289 (1949).

${ }^{2}$. Nature, 163, $602(1949)$.

\section{Shrinkage of Photographic Emulsions for Nuclear Research}

THE photographic emulsions used for the recording of ionizing particles contain a very high ratio of silver halide to gelatin. This results in a considerable shrinkage of the emulsion at processing, due to the washing out of the silver halide. In order to be able to interpret correctly events occurring in the emulsion, or to measure accurately the lengths of tracks which have a large angle of dip, it is important to know the exact relationship between the orientation of the track in the processed emulsion and the path of the particle while passing through the plate. This relationship is usually given by the so-called shrinkage factor $S$, which is the ratio of the thickness of the emulsion at exposure to that after processing; it can be determined either directly, by measuring the thicknesses of processed and unprocessed strips of emulsion, or by observations on tracks of known angle of dip $^{1}$ or known length ${ }^{2}$. The usual way to correct for shrinkage is to multiply the vertical projection of the track-length by the shrinkage factor. This is based on the assumption that the shrinkage affects all tracks in a uniform manner; but this is not immediately obvious, owing to the complicated phenomena which occur at processing. Taking into account that the photographic emulsion technique is becoming a method of high precision, it was thought worth while to study the shrinkage problem in greater detail.

First we determined the shrinkage factor, by direct measurement of thicknesses, in various types of emulsions with different loadings, concentrations of gelatin, thicknesses, and under different conditions of development, fixing and washing. Our results show that the shrinkage factor roughly follows, although is always smaller than, the ratio of the total volume of the emulsion to that of the gelatin. For emulsions of normal composition we found that Ilford $C .2, E .1$, $G .5$ and Kodak NT.1 $a, N T \cdot 2 a$ and $N T \cdot 4$ emulsions give nearly the same shrinkage factor, namely, $S=2 \cdot 7 \pm 0 \cdot 2$, the limits being extreme values and including measurements on emulsions from different batches. For lithium-loaded emulsions $S$ may go up to $3 \cdot 2$, due to the washing out of the lithium sulphate. We have also found that increasing the strength of the developer, or the time of fixation or washing, increases the shrinkage factor; but the changes in $S$ are less than 10 per cent even for wide variations of processing conditions.

Our main aim was to determine whether the shrinkage is uniform throughout the depth of the emulsion, and whether the same correction can be applied to all angles of dip. For this purpose we used mainly lithium-loaded emulsions (Ilford $C .2$ and $E .1$ ), which we exposed to a flux of thermal neutrons. In 